\section{RevistadePolítica Económica y Desarrollo Sostenible}

Centro Internacional de Política Económica para el Desarrollo Sostenible
Revista de Política Económica y Desarrollo Sostenible

EISSN: 2215-4167 • Vol. 2 (2) • Enero- Junio, 2017: 1-23

DOI: http://dx.doi.org/10.15359/peds.2-2.1

URL: http://www.revistas.una.ac.cr/politicaeconomica

Revista electrónica semestral publicada por el Centro Internacional de Política Económica para el Desarrollo Sostenible

Universidad Nacional, Lagunilla, Heredia Apartado 2393-3000

\title{
Actividades Socioeconómicas que emplean recursos naturales de la zona marítimo-terrestre y marina en Costa Rica y su relación con la variabilidad climática
}

\author{
Socio-economic activities using natural resources of the Marine and Terrestrial-Marine \\ Zone in Costa Rica and its relation to climate variability
}

Mary L. Moreno ${ }^{1}$, Roger Moya 2 y Eric J. Alfaro ${ }^{3}$

Fecha de recepción: 17. 10.2016 Fecha de aceptación: 04.04.2017 Fecha de publicación: 30.05.2017

\begin{abstract}
RESUMEN
La variabilidad climática podría tener efectos negativos sobre la productividad de las actividades que se desarrollan en las zonas costeras. Siendo Costa Rica un país en el que las actividades costeras relacionadas con recursos naturales tienen gran relevancia social y económica, en el presente artículo se realiza un análisis para verificar si estos efectos se presentan y brinda herramientas para la toma de decisiones en áreas costeras. Para lo anterior, se realiza una caracterización de la pesca y el sector turístico para el periodo 1990-2005. Se relaciona la cantidad de pesca tanto artesanal como industrial, la cantidad de turistas, hoteles y de habitaciones en zonas costeras con algunas variables climáticas con el fin de identificar las principales interacciones entre ellas. Las variables climáticas empleadas fueron: Temperatura Superficial del Mar (TSM) y Precipitación (P). Se emplearon coeficientes de correlación de Pearson entre las variables productivas y las climáticas. Al correlacionar las diferentes variables climáticas con la cantidad de pesca, se encontró que cuando el coeficiente fue estadísticamente significativo la variable climática en todos los casos tiene correlaciones negativas con las capturas; por ejemplo, cuando aumenta la TSM del Mar disminuye la cantidad de pesca. En el caso del turismo, los coeficientes no fueron estadísticamente significativos en las variables anuales pero sí en las trimestrales. La metodología aplicada en el presente artículo puede ser empleada para realizar inferencias sobre el comportamiento de las variables productivas para periodos específicos (trimestre por ejemplo) ante variaciones en climáticas y tomar medidas para mitigar el impacto.
\end{abstract}

Palabras Clave: Costa Rica, América Central, zona costera, turismo, pesca marina, climatología

\section{ABSTRACT}

Climate variability could have negative effects on the productivity of coastal zone activities. Because of the above and because Costa Rica is a country in which coastal activities, related to natural resources, have great social

1 Ph.D en Gestión de Recursos Naturales, por el programa interuniversitario del Instituto Tecnológico de Costa Rica, la Universidad Nacional, Costa Rica y la Universidad Estatal a Distancia (UNED). Docente- investigadora del Centro Internacional de Política Económica para el Desarrollo Sostenible (CINPE) Universidad Nacional, Costa Rica. ORCID ID: 0000-0003-0838-745X

2 Escuela de Ingeniería Forestal Instituto Tecnológico de Costa Rica, Cartago, Costa Rica. ORCID ID: 0000-0002-6201-8383

3 Centro de Investigaciones Geofísicas (CIGEFI), Escuela de Física y Centro de Investigación en Ciencias del Mar y Limnología (CIMAR), Universidad de Costa Rica. ORCID ID: 0000-0001-9278-5017 
and economic relevance, in the present article an analysis is made to verify if these effects are presented and to provide tools for making Decisions in these areas. For the above, a characterization of the fishing and the tourist sector for the period 1990-2005 is made. The amount of artisanal and industrial fishing is related, the number of tourists, hotels and rooms in coastal areas with some climatic variables in order to identify the main interactions between them. The climatic variables used were Sea Surface Temperature (SST) and Precipitation (P). Pearson correlation coefficients were used between productive and climatic variables. When correlating the different climatic variables with the amount of fishing it was found that when the coefficient was statistically significant, the climatic variable in all cases has negative correlations with the catches, that is to say when it increases for example the SST of the Sea decreases the amount of fishing. In the case of tourism, the coefficients were not statistically significant in the annual variables but in the quarterly ones. The methodology applied in this article can be used to make inferences about the behavior of productive variables for specific periods (quarter for example) in the face of climatic variations and to take measures to mitigate the impact.

Keywords: Costa Rica, Central America, coastal area, tourism, marine fisheries, climatology

\section{Introducción}

La zona marítimo-terrestre (ZMT) en Costa Rica se define como aquel territorio a 200 metros de la línea costera, cualquiera que sea su naturaleza, medidos horizontalmente a partir de la línea de la pleamar ordinaria y los terrenos y rocas que deje el mar en descubierto en la marea baja (Asamblea Legislativa, 1977). En la ZMT se desarrollan una gran cantidad de actividades económicas como pesca y turismo (Morales, Silva y González, 2010).

Sin embargo, estas zonas carecen de una visión técnica por parte de los entes regulares, lo que provoca que los recursos naturales sean vulnerables (Rojas, 2008). Por ejemplo, se señala que en las ZMT se solicitan concesiones sin cumplir los requisitos y los gobiernos locales no tienen un registro adecuado de las concesiones otorgadas, ni capacidad de fiscalizar el uso de las mismas (Román, 2009). Por otro lado, los cambios climáticos que afectan la intensidad de los ciclones y depresiones tropicales, el nivel del mar (Holgate y Woodworth, 2004) y la temperatura superficial del mar (Bindoff et al., 2007) producen variaciones en los ecosistemas marinos, la biodiversidad marina, la morfología de las costas y la infraestructura costera; por consiguiente, provocan un impacto social y económico en estas regiones (Lizano, 2001; Salazar-Vallejo 2002; Lizano y Lizano, 2010).

Además, según Parry et al. (2007), Schallenberg et al. (2003), Plagányi et al. (2011), OLDEPESCA (2010), Brenes (2010) y Samaniego (2009), el sector pesquero es afectado por variaciones en la temperatura superficial del mar. Además, Samaniego (2009) y Schatan et al. (2010) señalan que los aumentos en la temperatura y las disminuciones o aumentos en la precipitación influyen negativamente en las playas, la salud de los ecosistemas coralinos y la actividad turística.

En este sentido, de acuerdo con el Panel Intergubernamental de Cambio (IPCC, por sus siglas en inglés): 
Revista de Política Económica y Desarrollo Sostenible

EISSN: 2215-4167 • Vol. 2 (2) • Enero- Junio, 2017: 1-23

DOI: http://dx.doi.org/10.15359/peds.2-2.1

URL: http://www.revistas.una.ac.cr/politicaeconomica

Moreno, Moya y Alfaro

La variabilidad climática se refiere a las variaciones en el estado medio y otros datos estadísticos (como las desviaciones típicas, la ocurrencia de fenómenos extremos, etc.) del clima en todas las escalas temporales y espaciales, más allá de fenómenos meteorológicos determinados. La variabilidad se puede deber a procesos internos naturales dentro del sistema climático (variabilidad interna), o a variaciones en los forzamientos externos antropogénicos (variabilidad externa) (2013, p. 202).

Algunos estudios en Costa Rica mencionan los posibles efectos de la variabilidad climática sobre el sector pesquero y turístico. Por ejemplo, un estudio realizado por OLDEPESCA (2010) menciona que las alteraciones oceanográficas y atmosféricas ocurridas entre 1990 y 2005 impactaron al sector pesquero costarricense. Las capturas de tiburón (Carcharhinus falciformis) y dorado (Coryphaena hippurus) presentaron incrementos considerables y fueron atribuidas a los aumentos en la temperatura superficial del mar ocurridos durante el fenómeno de El Niño. Estos resultados se basan en la comparación del comportamiento de algunos fenómenos climáticos y las capturas pesqueras, sin realizar una verificación estadística.

Por otro lado, Schatan et al. (2010) y Samaniego (2009) mencionan que la costa del Pacífico Norte, en la provincia de Guanacaste de Costa Rica, enfrentará aumentos de temperatura de hasta $3.8{ }^{\circ} \mathrm{C}$ y una reducción en las precipitaciones de hasta un $63 \%$ en el 2100 y que estos cambios afectarán las actividades económicas. Esto provocará, según los anteriores autores, un impacto altamente negativo sobre la biodiversidad y la atracción turística de la región.

No obstante, a pesar de que se han presentado estos estudios en Costa Rica que asocian los efectos de las variables climáticas con las variables productivas en las actividades socioeconómicas relacionadas con recursos naturales en zonas costeras, dichos trabajos están basados en afirmaciones deductivas de otros estudios. Ante ello, el presente trabajo tiene como objetivo identificar los efectos de los cambios en las variables climáticas, precipitación y temperatura superficial del mar (TSM), sobre las actividades socioeconómicas desarrolladas en las zonas costeras en Costa Rica que emplean recursos naturales para su desarrollo.

\section{Materiales y métodos}

El estudio se llevó a cabo sobre la pesca y el turismo, actividades económicas relacionadas con la zona marítimo-terrestre (ZMT) y marina de Costa Rica que emplean recursos naturales para su desarrollo. Para obtener y analizar el impacto de las variaciones climáticas sobre las actividades productivas seleccionadas, se identificaron variables para cada una de las actividades y para la parte climática. En la figura 1 se muestran tanto la zona estudiada como los grupos de datos empleados para el análisis.

La información estadística de las capturas fue suministrada por INCOPESCA (2010) y se organizó por tipo de flota (industrial y artesanal) y por lugar de desembarque (Guanacaste, Golfo de Nicoya, Puntarenas, Quepos, Golfito y Limón). Posteriormente, se obtuvo los promedios anuales y trimestrales de estos datos. La organización de la información se realizó 
por trimestres debido a que algunos autores consideran que la información climática organizada de esta manera permite identificar cambios en el comportamiento de las variables climáticas en lapsos de tiempo cortos (Nienzy y Malone, 2003; Alfaro et al. 2010).

Para el sector turismo, se obtuvo una serie de datos del número de turistas extranjeros ( $\sin$ tendencia) que dijeron haber visitado playas en Costa Rica en el periodo 1990-2005 (ICT, 2002-2005). Con esta serie se calcularon los promedios anuales y los promedios trimestrales. En

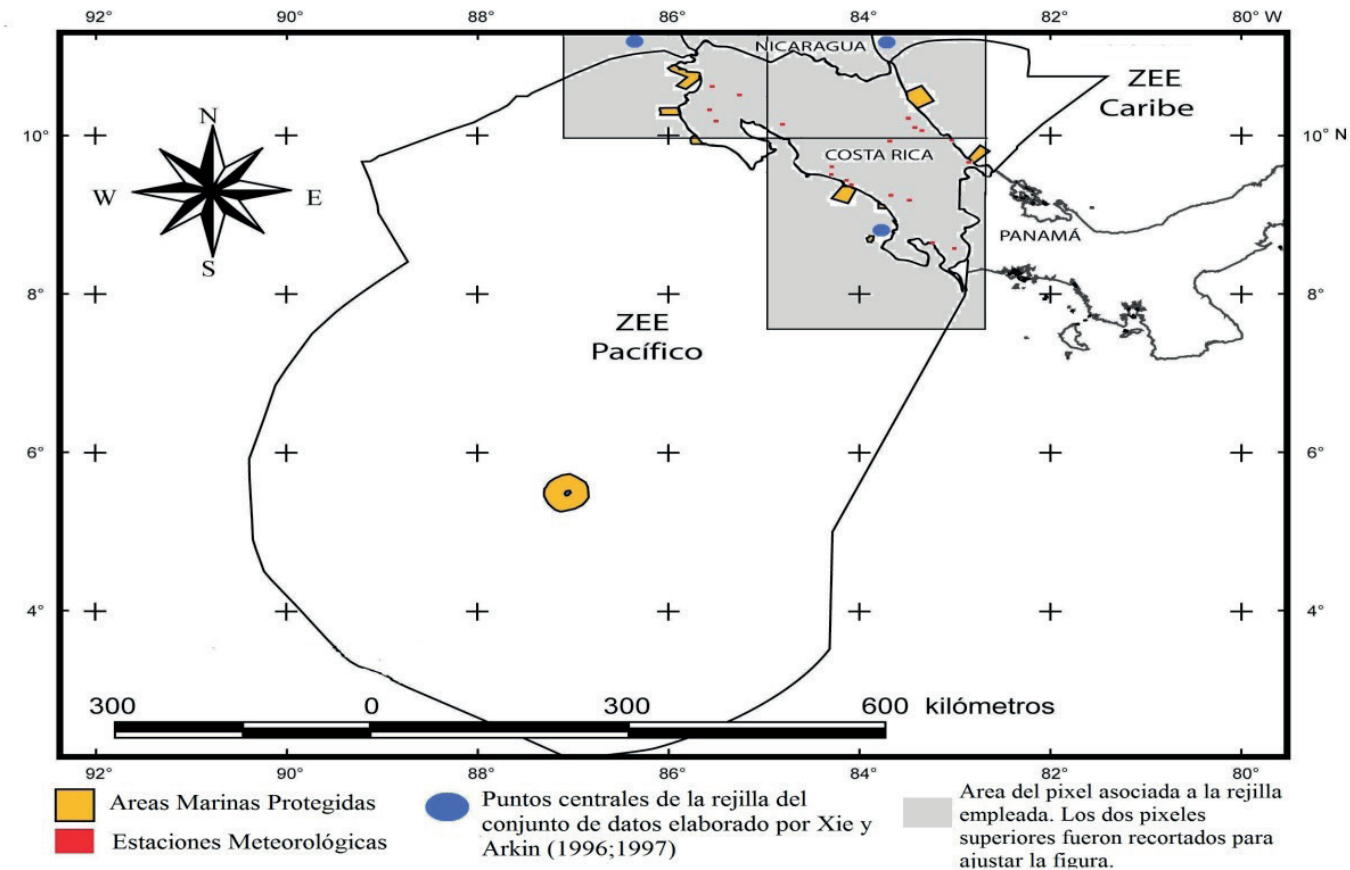

$\left.{ }^{*}\right)$ ZEE: Zona económica exclusiva.

Figura 1.Área terrestre y marítima de Costa Rica: áreas marinas protegidas

Fuente: Elaboración propia con información suministrada por SIGMAR (2005), Instituto Meteorológico Nacional (IMN), Instituto Costarricense de Electricidad (ICE), Xie y Arkin (1996,1997)

el caso de los datos de cantidad de hoteles y la cantidad de habitaciones en zonas costeras (ICT, 2011), también fueron calculados los promedios anuales y trimestrales.

Con respecto a las variables climáticas, se calcularon también los promedios anuales y trimestrales. La temperatura superficial del mar (TSM) se trabajó con el Índice Oceánico del Niño (ONI, por sus siglas en inglés), que se basa en las anomalías de dichas temperaturas y se define como una serie de promedios móviles trimestrales. A partir de estos datos, se define un evento de El Niño o cálido (La Niña o frío) si el índice es mayor o igual (menor o igual) a $0.5^{\circ} \mathrm{C}(-0.5$ 
Revista de Política Económica y Desarrollo Sostenible

EISSN: 2215-4167 • Vol. 2 (2) • Enero- Junio, 2017: 1-23

DOI: http://dx.doi.org/10.15359/peds.2-2.1

URL: http://www.revistas.una.ac.cr/politicaeconomica

Moreno, Moya y Alfaro

${ }^{\circ} \mathrm{C}$ ) durante al menos 5 trimestres móviles consecutivos (NOAA, 2011). Se obtuvo dos tipos de datos para la precipitación: datos de rejilla y datos de estaciones meteorológicas. Los datos de precipitación con base en datos de rejilla están referidos tanto a la región marina-costera como a la terrestre y se obtuvieron de la página WEB del International Research Institute for Climate and Society (IRI, 2011), que emplea la metodología de Xie y Arkin (1996; 1997). Estos datos están agrupados para tres regiones: Pacífico Norte, Pacífico Centro-Sur y Caribe y en total se trabajó con 768 datos. En el caso de las estaciones meteorológicas, se obtuvo los datos del Instituto Meteorológico de Costa Rica (IMN) y del Instituto Costarricense de Electricidad (ICE). Los datos se organizaron en 4 regiones: Caribe, Pacífico Norte, Pacífico Central y Pacífico Sur. La división climática anterior es la empleada por el IMN. Debido a que los datos se recolectan en forma diaria, inicialmente se contó con una base de 116.736 datos.

La relación de las diferentes variables climáticas con las variables seleccionadas del sector turístico (cantidad de turistas, cantidad de hoteles y cantidad de habitaciones) y pesquero (capturas en TM) se realizó por medio del coeficiente de correlación de Pearson, que permite saber si las variables están correlacionadas, la fuerza de esta relación (leve o estrecha) y si es positiva o negativa (Wilks, 2011). El valor de este coeficiente se encuentra entre 1 y -1 y el análisis de la significancia estadística se realiza con el valor- $p$ : si $\mathrm{p}<0.05$, el coeficiente es estadísticamente significante; pero si $\mathrm{p}>0.05$, el coeficiente no es estadísticamente significativo. Este coeficiente tiene dos grandes ventajas para el tipo de análisis que se realiza en este estudio. La primera es que el valor del coeficiente es independiente de cualquier unidad usada para medir variables, así se puede trabajar con variables climáticas y aquellas provenientes de actividades económica. La segunda es que entre más grande sea la muestra más exacta es la estimación y para el presente artículo se contó con bases de datos con gran cantidad y calidad de observaciones.

\section{Resultados}

El sector pesquero costarricense capturó 300054 TM en el periodo 1990-2005, de los cuales el 80.20 \% corresponde a pesca realizada por la flota artesanal y $19.80 \%$ a la flota industrial. La flota pesquera artesanal tuvo la mayor cantidad de capturas en Guanacaste, con aproximadamente el $55 \%$ del total de la pesca artesanal; luego destaca el Golfo de Nicoya con el 18 \%; y, posteriormente, Quepos (15\%), Golfito (9 \%) y Limón (3 \%) (Tabla 1 y Fig. 2), siendo este último sitio en el Mar Caribe. Para el caso de la flota industrial, a diferencia de la artesanal, se producen capturas solo en el pacífico (excepto en Golfito) y ninguna en el Mar Caribe. Los sitios con las mayores capturas se producen en el Golfo de Nicoya (55 \%), seguido por Quepos (24 \%) y Guanacaste (21 \%) (Tabla 1 y Fig. 3). Además, en el anexo 1 se presenta las capturas por año y mes para el periodo 1990-2005.

En referencia al sector turístico, durante el periodo 1990-2005 cerca de 15 millones de turistas extranjeros visitaron Costa Rica, el 50 \% (7.5 millones) dice tener la intención de visitar playas. En el anexo 2 se presenta las llegadas de turistas internacionales por mes por año. La 
cantidad total de visitantes extranjeros en el periodo evaluado varió entre 400 mil a 1.8 millones por año (Fig. 4 y 5 ).

En cuanto a las variables climáticas, la temperatura superficial del mar, representada por el ONI, varió entre - 0.9 y 1.3 (promedio anual), siendo el valor más bajo de - 0.9 en el año 1999 y el más alto en 1997, con un valor de 1.3. El análisis por trimestres arroja que en 59 valores de TSM (29\%) se presentaron eventos cálidos, en 49 (24\%) eventos fríos y en 94 (49\%) fueron considerado neutros.

Tabla 1

Costa Rica. Total de capturas por flota por región 1990-2005

(Toneladas métricas)

\begin{tabular}{ccccccccc}
\hline & \multicolumn{2}{c}{ Guanacaste } & \multicolumn{2}{c}{ Golfo de Nicoya } & \multicolumn{2}{c}{ Quepos } & Golfito & Caribe \\
\hline Año & $\begin{array}{c}\text { Pesca } \\
\text { artesanal }\end{array}$ & $\begin{array}{c}\text { Pesca } \\
\text { industrial }\end{array}$ & $\begin{array}{c}\text { Pesca } \\
\text { artesanal }\end{array}$ & $\begin{array}{c}\text { Pesca } \\
\text { industrial }\end{array}$ & $\begin{array}{c}\text { Pesca } \\
\text { artesanal }\end{array}$ & $\begin{array}{c}\text { Pesca } \\
\text { industrial }\end{array}$ & $\begin{array}{c}\text { Pesca } \\
\text { artesanal }\end{array}$ & $\begin{array}{c}\text { Pesca } \\
\text { artesanal }\end{array}$ \\
\hline 1990 & 7,780 & 1,438 & 2,483 & 1,594 & 2,207 & 1,436 & 978 & 640 \\
\hline 1991 & 5,961 & 449 & 2,254 & 2,367 & 1,634 & 629 & 508 & 433 \\
\hline 1992 & 5,020 & 736 & 2,589 & 2,018 & 1,616 & 1,140 & 431 & 261 \\
\hline 1993 & 5,483 & 635 & 2,300 & 1,418 & 1,401 & 723 & 475 & 199 \\
\hline 1994 & 6,307 & 836 & 2,077 & 1,385 & 1,299 & 763 & 643 & 268 \\
\hline 1995 & 6,610 & 800 & 2,026 & 1,190 & 1,187 & 873 & 923 & 422 \\
\hline 1996 & 7,315 & 4,142 & 2,176 & 1,340 & 2,128 & 973 & 1,639 & 436 \\
\hline 1997 & 10,080 & 389 & 2,584 & 1,879 & 2,878 & 1,074 & 2,264 & 421 \\
\hline 1998 & 8,011 & 434 & 3,423 & 1,648 & 1,866 & 742 & 1,788 & 363 \\
\hline 1999 & 9,072 & 584 & 4,510 & 2,433 & 2,232 & 800 & 1,378 & 665 \\
\hline 2000 & 13,646 & 277 & 3,053 & 2,006 & 2,806 & 889 & 2,087 & 1,051 \\
\hline 2001 & 14,298 & 252 & 2,244 & 2,542 & 3,872 & 813 & 2,398 & 681 \\
\hline 2002 & 11,523 & 304 & 2,440 & 3,171 & 3,651 & 1,033 & 2,140 & 622 \\
\hline 2003 & 10,512 & 305 & 2,651 & 2,726 & 2,307 & 955 & 657 & 557 \\
\hline 2004 & 5,508 & 323 & 2,999 & 2,536 & 2,548 & 637 & 820 & 535 \\
\hline 2005 & 5,226 & 427 & 3,279 & 2,579 & 2,922 & 751 & 1,443 & 541 \\
\hline
\end{tabular}

Fuente: Elaboración propia con base en datos de INCOPESCA (2011).

Por otro lado, los datos de precipitación anual obtenidos de los datos de rejilla se presentan en las figuras $4 \mathrm{a}$ y $4 \mathrm{~b}$. En estas se puede observar que la ZMT del Caribe presenta una precipitación mínima acumulada en 1991 de 781 mm y una máxima en 2002 de 1757 mm. Para el Pacífico Centro-Sur, el año con menor precipitación fue 2002 (145 mm) y el de mayor fue 1993 (381 mm). Asimismo, para el Pacífico Norte, el año con menor precipitación fue 2005 (805 mm) y el de mayor fue 1995 (1 $075 \mathrm{~mm}$ ). 


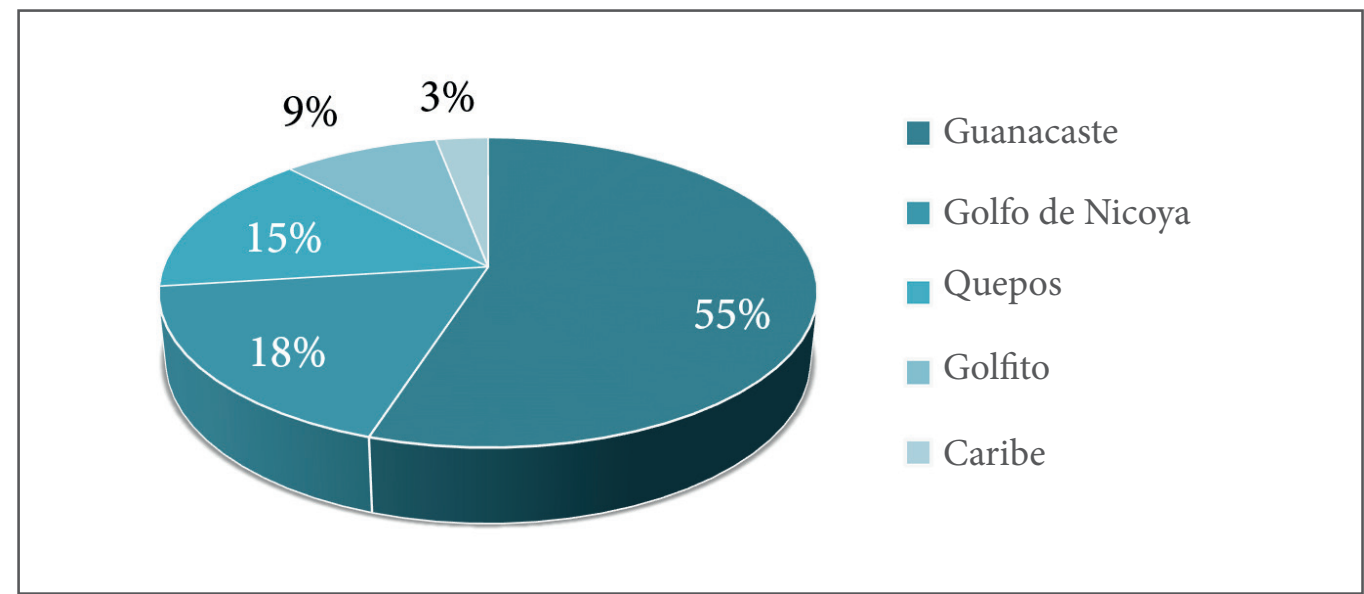

Figura 2.Costa Rica. Total de capturas de la flota artesanal por región, en porcentajes, -1990-2002-

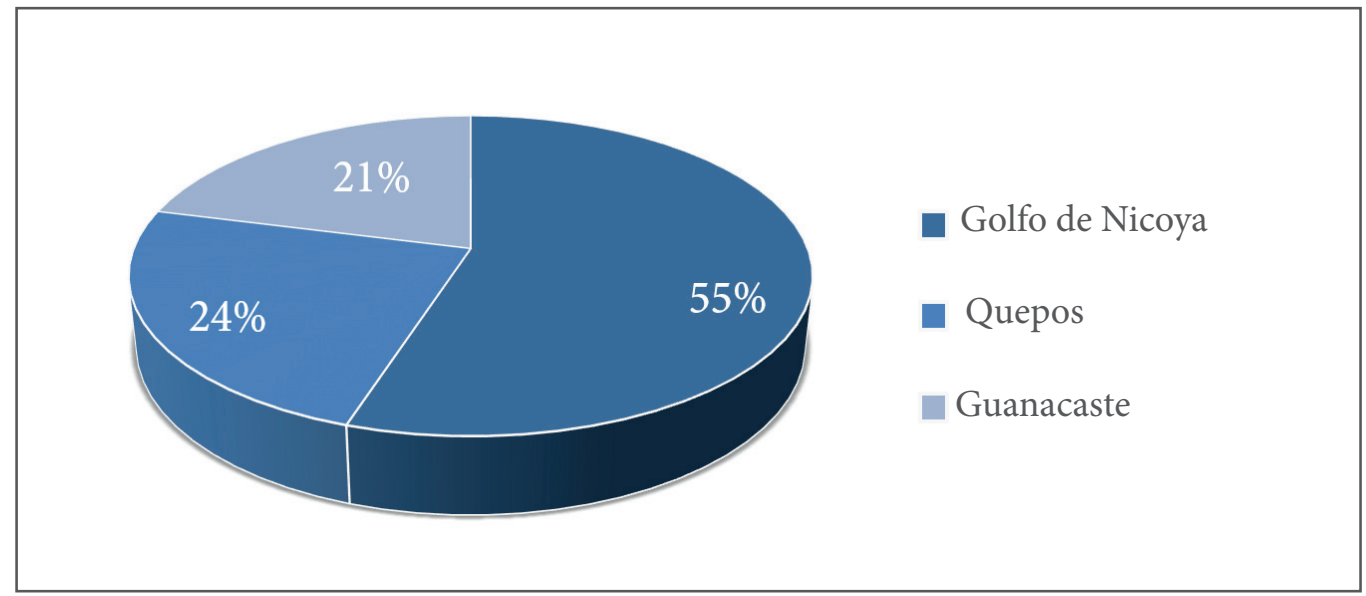

Figura 3. Costa Rica. Porcentaje de capturas por región de la flota industrial, 1990-2005 Fuente: Elaboración propia con base en INCOPESCA (2011). 
Revista de Política Económica y Desarrollo Sostenible

EISSN: 2215-4167 • Vol. 2 (2) • Enero- Junio, 2017: 1-23

DOI: http://dx.doi.org/10.15359/peds.2-2.1

URL: http://www.revistas.una.ac.cr/politicaeconomica

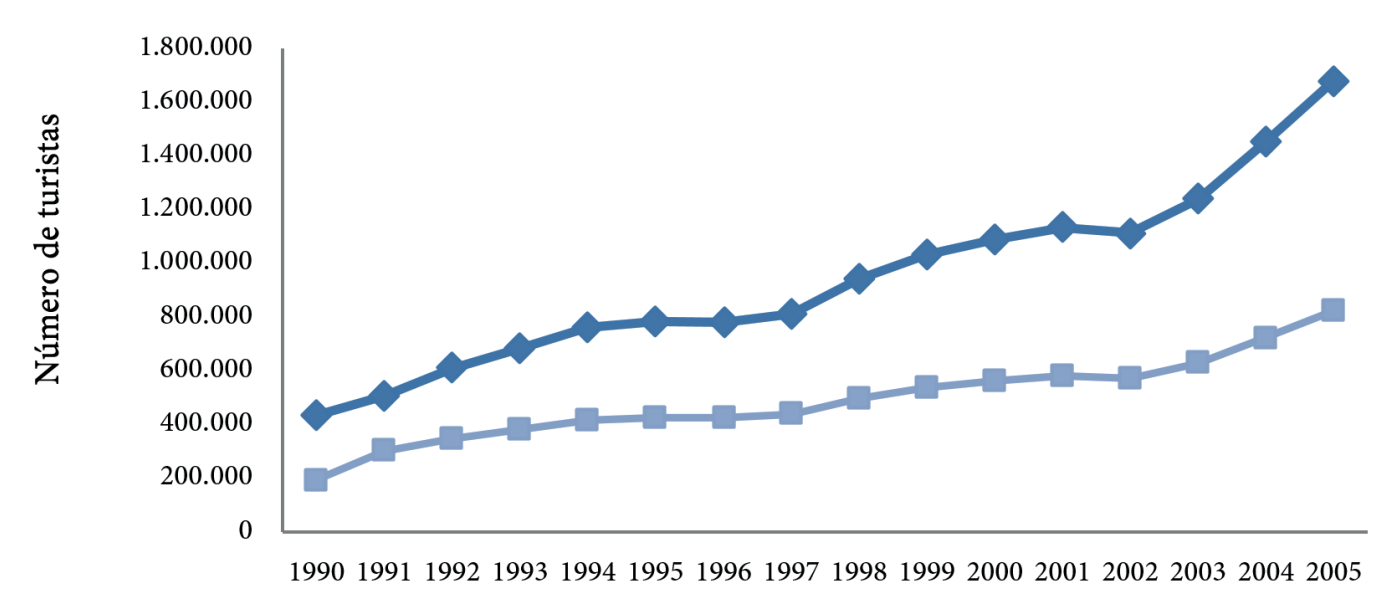

$\neg$ Cantidad de turistas extranjeros $\quad-\quad$ Extranjeros que realizan actividades de sol y playa

Figura 4. Costa Rica. Cantidad total de turistas extranjeros en comparación con la cantidad de turistas que visitan playas, 1990-2005

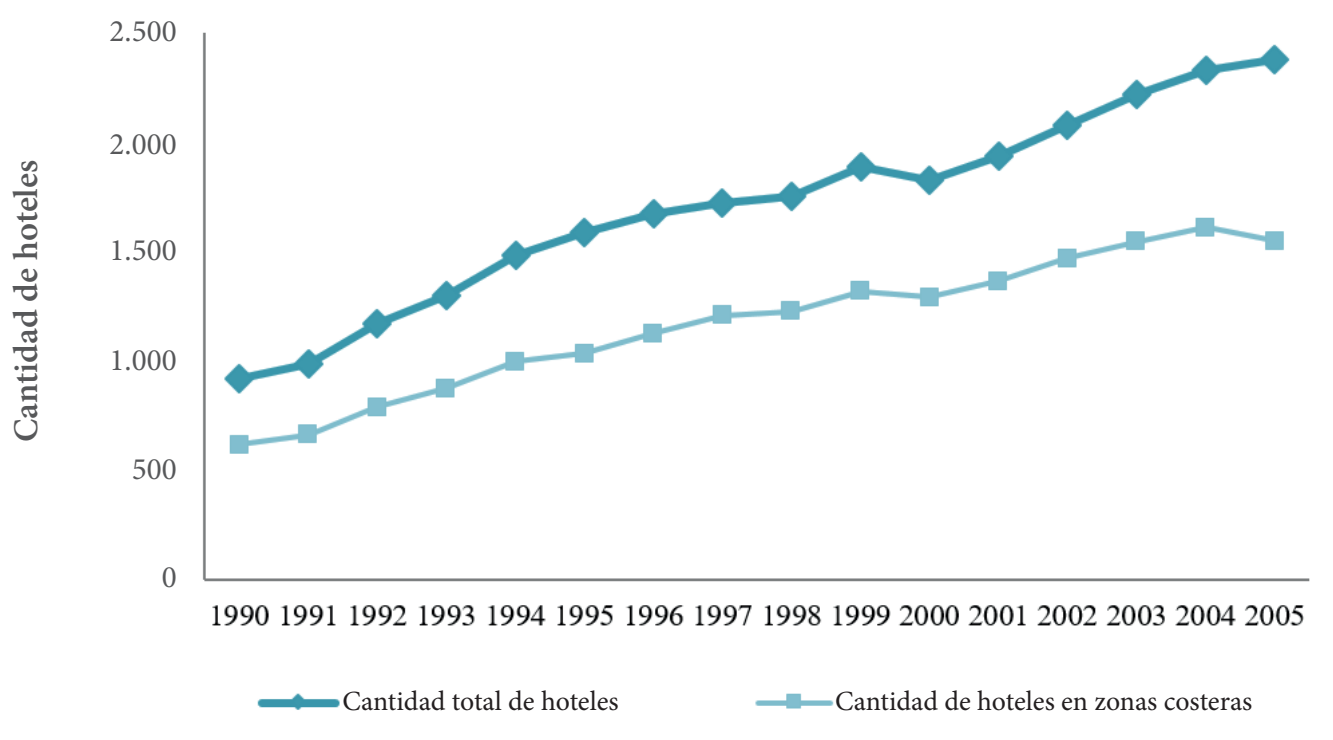

Figura 5. Costa Rica. Hoteles con y sin declaratoria turística en operación 1990-2005

Fuente: Elaboración propia con datos tomados de ICT, 1997-2005. 

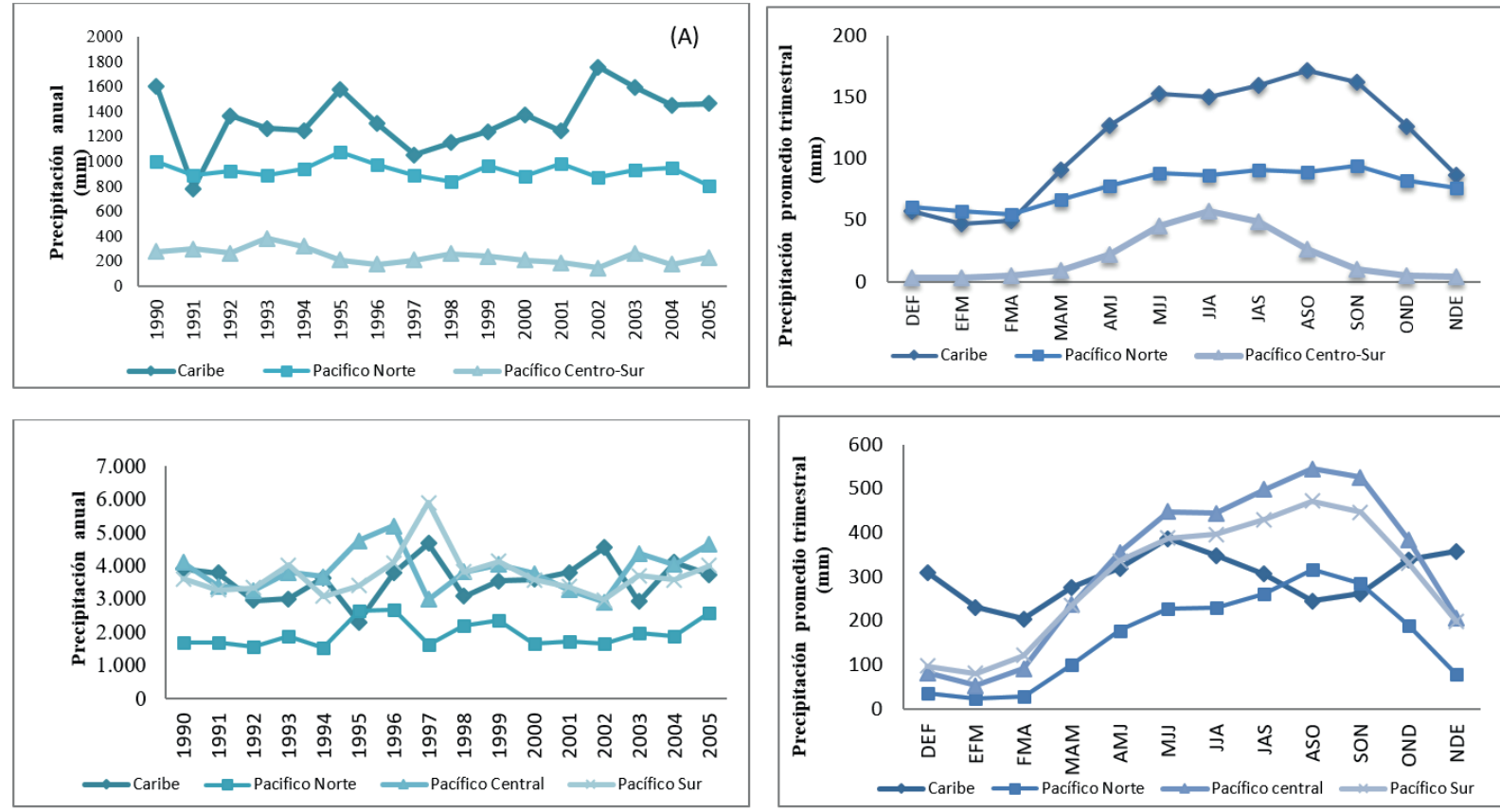

Figura 6. Costa Rica. Promedio de precipitación anual (a) y trimestral (b) empleando datos de rejilla y la precipitación anual (c) y trimestral (d) empleando las estaciones meteorológicas

Fuente: Elaboración propia con bases de datos obtenidos del IRI (2011)

Los datos de precipitación con base en estaciones meteorológicas se presentan en las figuras $6 \mathrm{c}$ y $6 \mathrm{~d}$. En estas se puede observar que para el Caribe el año con menor precipitación fue 1995 (2 296) mm y el de mayor fue 1997 (4 678 mm). Para el Pacífico Central, el año con menor precipitación fue 2002 (2 $915 \mathrm{~mm}$ ) y el de mayor fue 1996 (5 $203 \mathrm{~mm}$ ). Por su parte, para el Pacífico Sur el año de menor precipitación fue 2002 (2 978 mm) y el de mayor fue 1997 (5 904 $\mathrm{mm}$ ). Finalmente, en el Pacífico Norte el año con menor precipitación fue 1994 (1 $522 \mathrm{~mm}$ ) y el de mayor fue 1996 (2 $681 \mathrm{~mm})$.

Al relacionar las dos variables climáticas con las capturas, se encontró que cuando este coeficiente fue estadísticamente significante $(P<0.05)$, la variable climática en todos los casos tiene correlaciones negativas en las capturas (Cuadro 1). Así mismo, la TSM no fue correlacionada con las capturas anuales, pero esta variable climática sí fue negativamente correlacionada en el trimestre AMJ con las capturas totales, las del Pacífico y las del Caribe. Este mismo efecto fue encontrado (correlación negativa entre TSM y las capturas totales) en el trimestre MJJ de la región del Caribe (Cuadro 1).

En el caso de las precipitaciones, se encontró que la precipitación anual total de los datos de rejilla se correlaciona negativamente con las capturas totales, las capturas de la flota del 
Pacífico y especialmente las capturas artesanales de esta región de Costa Rica (Cuadro 1). Estas correlaciones negativas quedan evidenciadas cuando se analizan este tipo de datos para el Pacífico Central-Sur, pues nuevamente se ven afectadas de forma negativa las capturas totales, las capturas de la flota artesanal del Pacífico y las capturas totales de la costa Pacífica (Cuadro 1).

De igual forma, se encontró que las precipitaciones en los trimestres JJA y JAS presentan la mayor cantidad de correlaciones significantes con las capturas pesqueras. Específicamente en estos trimestres, la precipitación obtenida de datos de rejilla del Pacífico Central-Sur fueron estadísticamente relacionados con los diferentes tipos de capturas (total, flota artesanal del Pacífico y el total de capturas en la costa Pacífica y Caribe).

Cuadro 1.

Coeficientes de correlación (Pearson) entre las variables climáticas y las capturas pesqueras

\begin{tabular}{|c|c|c|c|c|c|c|}
\hline \multirow[t]{2}{*}{ Variable } & \multirow[t]{2}{*}{$\begin{array}{c}\text { Acumulado } \\
\text { anual }\end{array}$} & \multicolumn{5}{|c|}{$\begin{array}{c}\text { Promedio trimestral del acumulado } \\
\text { mensual }\end{array}$} \\
\hline & & AMJ & MJJ & JJA & JAS & ASO \\
\hline \multicolumn{7}{|c|}{$\begin{array}{l}\text { Temperatura superficial del mar-TSM } \\
\text { (Anomalía) }\end{array}$} \\
\hline Captura total & & -0.52 & & & & \\
\hline Captura total Pacífico & & -0.50 & & & & \\
\hline Total Caribe & & -0.58 & -0.52 & & & \\
\hline \multicolumn{7}{|c|}{ Precipitación total. Datos de rejilla } \\
\hline Captura total & -0.52 & & & -0.51 & -0.65 & \\
\hline Captura flota artesanal Pacífico & -0.50 & & & & -0.62 & \\
\hline Captura total Pacífico & -0.51 & & & -0.51 & -0.65 & \\
\hline \multicolumn{7}{|c|}{$\begin{array}{l}\text { Precipitación Pacífico Central-Sur. } \\
\text { Datos de rejilla. }\end{array}$} \\
\hline Captura total & -0.63 & & & -0.67 & -0.74 & -0.56 \\
\hline Captura flota artesanal Pacífico & -0.56 & & & -0.56 & -0.68 & \\
\hline Captura total Pacífico & -0.63 & & & -0.66 & -0.73 & -0.56 \\
\hline Captura total Caribe & & & & -0.69 & -0.71 & \\
\hline \multicolumn{7}{|c|}{ Precipitación Pacífico Norte. Estaciones meteorológicas } \\
\hline Captura flota artesanal & & & -0.52 & & & \\
\hline
\end{tabular}

Nota: Se presentan solamente los coeficientes que fueron significativos al $95 \%(P \leq 0.05)$. 
En el caso del sector de turismo, al relacionar las variables climáticas con la visitación anual turística, el número anual de hoteles y el número anual de habitaciones, se obtuvo que la TSM no se correlacionó significativamente con ninguna de las variables anteriores (Cuadro 2). La precipitación obtenida de los datos de rejilla afectó negativamente la construcción de hoteles en las zonas costeras (Cuadro 2). A su vez, la precipitación del Pacífico Central-Sur se correlacionó negativamente con el número de hoteles y habitaciones en esta zona (Cuadro 2). Un aspecto interesante de destacar de la visitación turística de sol y playa es que este está positivamente $(P \leq 0.05)$ correlacionado con la TSM, con la precipitación de datos de rejilla total y con la precipitación en el Pacífico Central-Sur medida en el trimestre JAS. Por último, el turismo de sol y playa se correlacionó positivamente con las precipitaciones del Caribe (datos de las estaciones meteorológicas) en el trimestre DEF (Cuadro 2).

Cuadro 2.

Coeficientes de correlación (Pearson) entre las variables climáticas y turismo de sol, hoteles y habitaciones en zonas costeras

\begin{tabular}{|c|c|c|c|c|}
\hline Variables & $\begin{array}{l}\text { Acumulado } \\
\text { anual }\end{array}$ & DEF & JAS & ASO \\
\hline \multicolumn{5}{|c|}{$\begin{array}{l}\text { Temperatura superficial del mar-TSM } \\
\text { (Anomalía) }\end{array}$} \\
\hline Turismo sol y playa & & & 0.57 & \\
\hline \multicolumn{5}{|l|}{$\begin{array}{l}\text { Precipitación total. } \\
\text { Datos de rejilla }\end{array}$} \\
\hline Turismo sol y playa & & & 0.67 & \\
\hline Hoteles zonas costeras & -0.64 & & & \\
\hline \multicolumn{5}{|c|}{$\begin{array}{l}\text { Precipitación Pacífico Central- Sur. } \\
\text { Datos de rejilla }\end{array}$} \\
\hline Turismo sol y playa & & & 0.79 & 0.73 \\
\hline Hoteles zonas costeras & -0.59 & & & \\
\hline Habitaciones zonas costeras & -0.58 & & & \\
\hline \multicolumn{5}{|l|}{$\begin{array}{l}\text { Precipitación del Caribe. } \\
\text { Estaciones meteorológicas. }\end{array}$} \\
\hline Turismo sol y playa & & 0.55 & & \\
\hline
\end{tabular}

Nota: Se presentan solamente los coeficientes que fueron significativos sobre $95 \%(\alpha \leq 0.05)$. 
Revista de Política Económica y Desarrollo Sostenible

EISSN: 2215-4167 • Vol. 2 (2) • Enero- Junio, 2017: 1-23

DOI: http://dx.doi.org/10.15359/peds.2-2.1

URL: http://www.revistas.una.ac.cr/politicaeconomica

Moreno, Moya y Alfaro

\section{Discusión}

En los resultados obtenidos de la serie 1990-2005 se tiene que la flota pesquera artesanal realiza más capturas que la industrial, con $80.20 \%$ y $19.80 \%$, respectivamente. Asimismo, fue comprobado que las capturas de la flota artesanal fueron creciendo a través de los años (Fig. 4). Lo anterior puede ser explicado debido a que la flota artesanal pequeña y mediana constituye cerca del $93 \%$ del total de embarcaciones y el $80 \%$ del total de capturas de la pesca comercial en el país (INCOPESCA, 2010). Las capturas de la flota artesanal en Costa Rica son consideras como de bajo aprovechamiento y poca rentabilidad, por lo que se tiene que tener capturas mayores para lograr beneficios (Víquez y Gutiérrez, 2005; Víquez y Sierra, 2005).

En lo relacionado con la visitación turística en Costa Rica, esta tuvo una tendencia creciente en todo el periodo de estudio, 1990-2005 (Fig. 6a). Lo anterior se debe principalmente al aumento en el nivel de inversiones de origen extranjero, en los litorales costarricenses a partir del 2001, contribuyendo a aumentar la oferta turística, especialmente en la ZMT de Guanacaste y Puntarenas (Rojas, 2008). En estas zonas se encuentran resorts con todo incluido y casas de vacaciones (Honey et al. 2010), debido mayormente a la belleza de las playas y la existencia de ecosistemas, como corales y manglares, muy atractivos para el turismo internacional (Morales et al. 2010).

Por otro lado, el análisis del comportamiento de las variables climáticas durante el periodo permite afirmar que los análisis de las series trimestrales de la precipitación y la TSM proporcionan un mayor detalle de los cambios en estas variables que las series anuales. Por ejemplo, considerando el promedio anual, se encontró que los años 1990, 1993, 1995, 1996, 1998, 2001, 2003 y 2005 no fueron considerados de Fenómeno de El Niño o Fenómeno de La Niña. No obstante, a excepción del año 1990, se presentaron eventos de El Niño (La Niña) en algunos de los trimestres de estos años. Otro ejemplo es el año 1995, con un ONI anual de 0.08 considerado como normal, en los trimestres DEF, EFM y FMA se presentó evento de El Niño, pero en los trimestres JAS, ASO, SON, OND y NDE se presentó evento de La Niña. Se debe mencionar que este tipo de análisis es desarrollado también en otros estudios relacionados con variables climáticas (Alfaro, 2007), aunque en algunos además se trabaja con datos mensuales en lugar de trimestrales (Amador,Alfaro, Rivera y Calderón, 2010).

Al relacionar la TSM con las capturas, se obtuvo que esta tiene correlaciones negativas sobre las capturas totales, las del Pacífico y las del Caribe para dos trimestres, AMJ y MJJ (Cuadro 1). En el caso del Pacífico - de acuerdo con Brenes (2010)-, la ocurrencia del fenómeno de El Niño provoca aumento en la temperatura superficial del mar, lo cual causa cambios negativos en la estructura del Pacífico Centroamericano y, como resultado, provoca afectaciones en la productividad de la región que repercuten en cambios en la abundancia y distribución de las poblaciones de peces y camarones. Caso contrario ocurre cuando se presenta el fenómeno de La Niña, ya que la productividad de los afloramientos costeros aumenta.

Como se muestra en la Cuadro 1, la precipitación de datos de rejilla se correlacionó negativamente con las capturas pesqueras para los totales y tres de las flotas (artesanal Pacífico, 
Revista de Política Económica y Desarrollo Sostenible

EISSN: 2215-4167 • Vol. 2 (2) • Enero- Junio, 2017: 1-23

DOI: http://dx.doi.org/10.15359/peds.2-2.1

URL: http://www.revistas.una.ac.cr/politicaeconomica

total Pacífico y total Caribe), tanto para las capturas anuales como para todas las flotas en el trimestre JAS y en el trimestre JJA (excepto para las capturas de la flota artesanal del Pacífico). Lo anterior podría indicar que de las variaciones de la precipitación de datos de rejilla que se producen en el año, no todas tienen efectos negativos en la pesca, solo las ocurridas en los trimestres JJA y JAS. Esto concuerda con el hecho de que para el periodo de análisis uno de los trimestres más lluviosos fue JJA (con $99.7 \mathrm{~mm}$ de promedio anual para este trimestre). Según el trabajo realizado por Chang y Del Rio (2004) en el Golfo de Nicoya, los pescadores asocian la lluvia con una disminución en los volúmenes de pesca. Se ha demostrado que, cuando aumenta las precipitaciones en las zonas costeras por efectos, por ejemplo, de los huracanes, uno de los sectores afectados negativamente es el pesquero (IMN, 2011).

Por último, la variable climática precipitación (medida en las estaciones meteorológicas) tuvo correlaciones negativas en el caso del Pacífico Norte sobre las capturas de la flota artesanal (Cuadro 1). Este resultado es muy importante ya que en esta zona se concentra la mayor cantidad de capturas de la flota artesanal (Fig. 2). El uso de las estaciones meteorológicas permite analizar con mayor precisión la relación entre la precipitación y las capturas, que los datos de precipitación de datos de rejilla, ya que éstas últimas abarcan un área más grande, como se muestra en la figura 1.

Al realizar el análisis de las variables climáticas y las variables de la actividad turística (Turismo de sol y playa, hoteles en zonas costeras y habitaciones en zonas costeras), se obtuvo que aumentos en la temperatura superficial de mar en el trimestre JAS tienen una correlación positiva con la cantidad de turistas que visitan las playas (Cuadro 2). Por otro lado, la mayor cantidad de relaciones se dieron con la variable precipitación de datos de rejilla, que tuvo efectos negativos en el promedio anual de hoteles de zonas costeras y habitaciones en zonas costeras (Cuadro 2), lo cual es previsible teniendo en cuenta que en los meses de mayor precipitación hay menor cantidad de turismo y el número de construcciones puede disminuir también.

La discusión presentada en los párrafos anteriores permite identificar las principales ventajas de realizar análisis como los que se presentan en el presente artículo. Por un lado, se logra cuantificar relaciones entre variables productivas y variables climáticas (Cuadros 1 y 2) que hasta el momento se habían realizado solamente por observación cualitativa del comportamiento de los datos. Por otro lado, es posible realizar inferencias sobre el comportamiento de las variables productivas para periodos específicos (trimestres por ejemplo), conociendo la evolución histórica de su relación con las variables climáticas. Estas comprobaciones estadísticas llegan a confirmar afirmaciones dadas, por ejemplo, por Brenes (2010), quien menciona que fenómeno de El Niño (evento cálido) puede afectar los recursos pesqueros a través del efecto del mismo sobre la calidad de las aguas que emergen durante los afloramientos. Estas aguas, además de ser muy cálidas, contienen mucho menos cantidad de nutrientes y la productividad primaria desciende substancialmente. Lo anterior se traduce en una disminución de las capturas pesqueras, en especial para algunas especies.

Aunque los resultados presentados en nuestro estudio en su mayoría son correlaciones negativas, otro estudios específicos, pero de tipo descriptivo, como el realizado por OLDEPESCA 
(2010), mostraron que el aumento de temperaturas por eventos cálidos (fenómeno de El Niño) en los años 1997-1998 y 2000-2001 favorecen las capturas de jaquetón (una de las especies más capturadas entre 1990 y 2004 en el país), debido a la incursión de aguas cálidas. Así mismo, otra especie que se vio favorecida fue la Lumpuga (Dorado) que presentó un gran incremento en las capturas a partir del año 1991, posiblemente gracias al ingreso de aguas cálidas por el desarrollo del evento cálido durante ese año.

La diferencia del análisis realizado en los artículos mencionados anteriormente y los resultados obtenidos en el presente trabajo consiste en que los primeros se basan en la observación de las variables climáticas y las capturas para realizar sus conclusiones, mientras que esta investigación establece relaciones estadísticas entre ellas, lo que permite de alguna manera cuantificar las posibles relaciones que ayuden a explicar los efectos de la variabilidad climática sobre los recursos naturales en las ZMT.

Por otro lado, es posible realizar inferencias sobre el comportamiento de las variables productivas de la ZMT para periodos específicos conociendo la evolución histórica de su relación con las variables climáticas. En el caso de la relación entre capturas totales y la temperatura superficial del mar (Cuadro 1), por ejemplo, se puede observar que hay una correlación negativa entre ellas, es decir, un aumento de la TSM estaría correlacionado con una disminución en las capturas en el trimestre AMJ y viceversa.

\section{Conclusiones}

Las principales actividades socioeconómicas desarrolladas en zonas costeras en Costa Rica que emplean recursos naturales como insumos para su desarrollo son la pesca y la actividad turística. Estas actividades han sido muy dinámicas en el periodo en estudio 1990-2005 y son muy importantes para las comunidades costeras. Las principales variables climáticas que se relacionan con estas actividades son la precipitación y la TSM.

En el periodo 1990-2005, el sector pesquero costarricense desembarcó 300054 TM de productos pesqueros en las cinco regiones para las flotas artesanal e industrial, produciéndose las mayores capturas en la región de Guanacaste. Al relacionar las diferentes variables climáticas con las capturas se encontró que cuando este coeficiente fue estadísticamente significativo $(P \leq 0.05)$, la variable climática en todos los casos tiene correlaciones negativas con las capturas. Así mismo, la TSM se correlacionó negativamente con las capturas totales del Pacífico y los del Caribe en el trimestre AMJ. En el caso de las precipitaciones, se encontró que la precipitación de datos de rejilla promedio y la del Pacífico Central-Sur de todo el país afectan de manera negativa las capturas totales, las capturas de la flota Pacífico y especialmente las capturas artesanales de esta región de Costa Rica.

Además, se encontró que en los trimestres JJA y JAS se presentan la mayor cantidad de correlaciones significativas con las capturas pesqueras. Específicamente en estos trimestres, la precipitación de datos de rejilla del Pacífico Central-Sur y la precipitación de datos de rejilla total 
de Costa Rica fueron estadísticamente relacionadas con los diferentes tipos de capturas (total, flota artesanal del Pacífico y el total de las capturas en la misma costa y en el Caribe).

En el caso del sector de turismo, se obtuvo que la TSM no se correlacionó significativamente con la visitación turística, el número de hoteles o el número de habitaciones anuales. Por otro lado, la precipitación de datos de rejilla total sí mostró correlaciones negativas. A su vez, la precipitación de datos de rejilla del Pacífico Central-Sur se correlaciona negativamente en la construcción de hoteles y habitaciones en esta zona. Un aspecto interesante de destacar del turismo es que este está positivamente $(P \leq 0.05)$ relacionado con la TSM, con la precipitación de datos de rejilla total y con la precipitación en el Pacífico Central y Sur medida en el trimestre JAS. Así, el turismo de sol y playa que fue afectado positivamente por las precipitaciones del Caribe fue el del trimestre DEF.

\section{Agradecimientos}

Mary Luz Moreno agradece al "Programa integrado de análisis de políticas públicas para la gestión sostenible de los recursos naturales y servicios ambientales en Costa Rica", del Centro Internacional de Política Económica para el Desarrollo Sostenible (CINPE) de la Universidad Nacional, por el apoyo brindado en tiempo y recursos.

Eric Alfaro le agradece a los siguientes proyectos de la UCR que le suministraron tiempo y recursos: 805-B7-507, B6-143 (ambos apoyados por CONICIT-MICITT), B4-227, B0-065, A9-532 (apoyado por CSUCA-ASDI), B5-298 y B0-810.

Róger Moya agradece el apoyo de la Vicerrectoría de Investigación y Extensión del Instituto Tecnológico de Costa Rica (ITCR), con quienes está muy agradecido por su apoyo a través de todas las fases de la elaboración del estudio.

\section{Referencias bibliográficas}

Alfaro, E., Quesada, A. \& Solano, F. (2010). Análisis del Impacto en Costa Rica de los Ciclones Tropicales ocurridos en el Mar Caribe desde 1968 al 2007. Diálogos Rev. Electr. Hist., 11(2), 22-38. Recuperado de https://revistas.ucr.ac.cr/index.php/dialogos/article/view/578/640

Alfaro, E. (2007). Escenarios climáticos para temporadas con alto y bajo número de huracanes en el Atlántico. Revista de Climatología, (7), 1-13.. Recuperado de: http://webs.ono.com/ reclim2/reclim07a.pdf

Amador, J. A., Alfaro, E., Rivera, E. \& Calderón, B. (2010). Climatic Features and Their Relationship with Tropical Cyclones Over the Intra-Americas Seas. En J.B. Elsner et al. (Eds.), Hurricanes and Climate Change (pp. 149-173). New York, EE. UU.: Springer. https:// doi.org/10.1007/978-90-481-9510-7 9 
Asamblea Legislativa. (1977). Ley No. 6043. Ley Sobre la Zona Marítimo Terrestre. La Gaceta, 52.

Brenes, C. (2010). El fenómeno del niño 2009-2010: Pacífico centroamericano y pesquerías. UNA-OSPESCA. Recuperado de http://www.google.co.cr/url?sa=t\&rct=j\&q=\&esrc=s\&source $=$ web\&cd=1\&ved=0CBwQFjAA\&url=http\%3A\%2F\%2Fwww.sica.int $\% 2$ Fbusqueda\%2Fbusqueda archivo.aspx\%3FArchivo\%3Dodoc $41706 \quad 1 \quad 02102009$.pdf\&ei=0TOQUI mLefL0QHvz4CgBg\&usg=AFQjCNEJz mEDYn20Enk4cW5Wk1Y7KCCFQ

Chang, G. \& Del Rio, X. (2004). Léxico de la Pesca Artesanal Puntarenense en el Golfo de Nicoya: Una aproximación etnográfica-semántica en Chomes, Costa de Pájaros, Isla Chira y Puntarenas Centro. Recuperado de http://www.vinv.ucr.ac.cr/latindex/kanina001/14-chang-delrio.pdf

Honey, M., Vargas, E. \& Durham, W. (2010). Impacto del Turismo Relacionado con el Desarrollo en la Costa Pacífica Costa Rica. Washington D.C., EE.UU.: Center for Responsible Travel.

ICT (2011). Hoteles en Costa Rica por Región. Instituto Costarricense de Turismo. Recuperado de http://www.visitcostarica.com/ict/paginas/buscador/fcrbuscaprod.asp?idprod=1].

ICT. (2002-2005). Anuario de Turismo. Instituto Costarricense de Turismo. San José, Costa Rica: ICT

IMN. (2011). Los Huracanes y sus efectos en Costa Rica. Instituto Meteorológico Nacional (IMN). Recuperado de [http://www.imn.ac.cr/educacion/huracanes/huracan06.html].

INCOPESCA. (2011). Estadísticas pesqueras 1990-2005. Bases de datos. Instituto Costarricense de Pesca. San José, Costa Rica: INCOPESCA.

IPCC. (2013). Glosario [Planton, S. (ed.)]. En: Cambio Climático 2013. Bases físicas. Contribución del Grupo de trabajo I al Quinto Informe de Evaluación del Grupo Intergubernamental de Expertos sobre el Cambio Climático [Stocker, T.F., D. Qin, G.-K. Plattner, M. Tignor, S.K. Allen, J. Boschung, A. Nauels, Y. Xia, V. Bex y P.M. Midgley (eds.)]. Cambridge University Press, Cambridge, Reino Unido y Nueva York, NY, Estados Unidos de América.

IRI. (2011). Climate Data Library. Recuperado de http://iridl.ldeo.columbia.edu/index.html

Morales, A., Silva, M. \& González, C. (2010). La Gestión Integrada de la Zona Costera en Costa Rica: Experiencias y Perspectivas. En J. M. Barragán (coord.), Manejo Costero Integrado y Política Pública en Iberoamérica: Un diagnóstico. Necesidad de Cambio (pp. 41-70). Cádiz, España: Red IBERMAR (CYTED).

Nienzy, B. \& Malone, L. (2003, diciembre). Desafíos de la predicción climática y su aplicación en el sector Agrícola. Ponencia presentada en la Reunión Técnica de la Organización Meteorológica Mundial. Servicios de Información y Predicción del Clima (SIPC) y Aplicaciones Agrometeorológicas para los Países Andinos. Guayaquil, Ecuador. 
NOAA. (2011). Changes to the Oceanic Niño Index (ONI). Recuperado de disponible en http:// www.cpc.noaa.gov/data/indices/sstoi.atl.indices

OLDEPESCA. (2010). Efectos de las principales alteraciones atmosféricas y oceanográficas sobre la actividad pesquera de los países miembros de OLDEPESCA. San Francisco de Campeche, México: Organización Latinoamericana de Desarrollo Pesquero.

Parry, M., Canziani, O., Palutikof, J., Van der Linden P., \& Hanson, C. (2007). Climate Change 2007: Impacts, Adaptation and Vulnerability. Contribution of Working Group II to the Fourth Assessment Report of the Intergovernmental Panel on Climate Change. Cambridge University Press, Cambridge, United Kingdom and New York. NY, EE.UU. pp.507-540. Recuperado de http://www.ipcc.ch/pdf/assessment-report/ar4/wg2/ar4 wg2 full report.pdf

Plagányi, E., Weeks, S., Skewes, T., Gibbs, M., Poloczanska, E., Norman-López, A., Blamey, L., Soares, M. \& Robinson, W. (2011). Assessing the adequacy of current fisheries management under changing climate: a southern synopsis, ICES. J. Mar. Sci. 6(68) 1305-1317. https://doi.org/10.1093/icesjms/fsr049

Rojas, A. (2008). Sitio y Situación de la Zona Marítimo Terrestre del Pacífico y Caribe Costarricense. Reflexiones, 87(1), 163-176.

Samaniego, J. (2009). Cambio Climático y desarrollo en América Latina y el Caribe: Una reseña. Santiago de Chile, Chile: CEPAL.

Schallenberg, M., Hall C. J. \& Burns, C. W. (2003). Consequences of climate-induced salinity increases on zooplankton abundance and diversity in coastal lakes. Mar. Ecol. Prog. Ser., 251. 181-189. https://doi.org/10.3354/meps251181

Schatan, C., Montile, M. \& Romero, I. (2010). Cambio climático y retos para el sector turismo de Centroamérica. Santiago de Chile, Chile: CEPAL.

SIGMAR (2005). Base de datos áreas marinas protegidas de Costa Rica. Proyecto: Desarrollo de un Sistema de Información Geográfica en el Centro de Investigación en Ciencias del Mar y Limnología (CIMAR). Universidad de Costa Rica.

Víquez, R. \& Gutiérrez, A. (2005). Comportamiento de la Almadraba Japonesa en Tárcoles. Ambientico, 139. 15-17.

Víquez, R. \& Sierra, L. (2005). Pesca Artesanal en Tárcoles, Costa Rica. Ambientico, 139. 13-14.

Wilks, D. (2011). Statistical Methods in the Atmospheric Sciences. San Diego, CA., EE. UU.: Academic Press.

Xie, P. \& Arkin, P. A. (1996). Analyses of Global Monthly Precipitation Using Gauge Observations, Satellite Estimates, and Numerical Model Predictions. J. Climate, 9. 840-858. https://doi. org/10.1175/1520-0442(1996)009<0840:AOGMPU>2.0.CO;2 
Xie, P. \& Arkin, P. A. (1997). Global Precipitation: A 17-Year Monthly Analysis Based on Gauge Observations, Satellite Estimates and Numerical Model Outputs. Bull. Amer. Meteor. Soc., 78. 2539-2558. https://doi.org/10.1175/1520-0477(1997)078<2539:GPAYMA $>2.0 . C O ; 2$

Anexo 1. Costa Rica. Capturas totales por mes, 1995-2005

\begin{tabular}{|c|c|c|c|c|c|c|c|c|c|c|c|c|}
\hline Año & Enero & Febrero & Marzo & Abril & Mayo & Junio & Julio & Agosto & Septiembre & Octubre & Noviembre & Diciembre \\
\hline 1990 & $1.866,6$ & $1.674,4$ & $1.627,2$ & $1.949,6$ & $1.780,6$ & $1.213,3$ & $1.279,8$ & $1.358,4$ & $1.517,3$ & $1,305,7$ & $1.534,4$ & $1.448,6$ \\
\hline 1991 & $1.576,5$ & $1.110,6$ & $1.310,2$ & $1.014,4$ & 970,7 & $1.060,2$ & 953,0 & $1.229,1$ & $1.172,2$ & $1.238,1$ & $1.263,8$ & 1 \\
\hline 1992 & $1.177,3$ & 986,7 & $1.196,1$ & $1.591,6$ & $1.058,5$ & $1.225,0$ & $1.145,8$ & $1.236,5$ & $1.129,5$ & $1.074,8$ & $1.109,1$ & \\
\hline 1993 & 901,9 & 954,4 & $1.059,7$ & $1.106,2$ & $1.047,4$ & $1.070,3$ & $1.079,7$ & $1.139,6$ & $1.014,8$ & $1.098,7$ & $1.077,8$ & 1.084 \\
\hline 1994 & 784,9 & 938,2 & $1.163,6$ & $1.004,4$ & $1.151,9$ & $1.241,8$ & $1.153,3$ & $1.284,5$ & $1.136,9$ & $1.135,4$ & $1.266,3$ & $1.317,4$ \\
\hline 1995 & $1.064,2$ & $1.053,5$ & $1.132,8$ & $1.014,7$ & $1.245,2$ & $1,344,8$ & $1.168,1$ & $1.175,6$ & $1.073,4$ & $1.091,6$ & $1.269,6$ & 1.397 \\
\hline 1996 & $1.308,3$ & $1.821,6$ & $1,307,0$ & $2,107,8$ & $1.226,6$ & $1.274,2$ & $2.306,9$ & $2.489,7$ & $1.414,2$ & $1,396,2$ & $1,617,5$ & $1.878,9$ \\
\hline 1997 & $2.020,5$ & $1.807,9$ & $1.824,6$ & $1.717,5$ & $1.620,3$ & $1.595,3$ & $2.273,6$ & $1.972,8$ & $1.830,6$ & $1.459,0$ & $1,626,8$ & 1.81 \\
\hline 1998 & $1.475,0$ & $1.337,2$ & $1.640,8$ & $1.613,0$ & $1.616,2$ & $1.612,5$ & $1.486,8$ & $1.737,9$ & $1.392,6$ & $1.335,8$ & $1.556,4$ & $1.4 / 6$ \\
\hline 1999 & $1.520,6$ & $1.403,0$ & $1.383,9$ & $1.467,3$ & $1.939,3$ & $1.812,8$ & $1.863,5$ & $1.272,0$ & $1.739,6$ & $1.888,2$ & $2.514,7$ & 286 \\
\hline 2000 & $1.965,1$ & $2.276,8$ & $1.943,4$ & $1.695,9$ & $1.921,7$ & $1.875,9$ & $2.090,6$ & $2.167,3$ & $2.053,6$ & $2.311,7$ & $2.594,3$ & 2.919 \\
\hline 2001 & $2.509,0$ & $2.083,2$ & $2.403,1$ & $1.994,4$ & $2.642,9$ & $1.963,8$ & $2.145,8$ & $1.971,4$ & $1.983,8$ & $2.290,0$ & $2.697,9$ & 2.41 \\
\hline 2002 & $2.030,8$ & $2.239,7$ & $1.910,6$ & $1.632,6$ & $1.790,2$ & $1.805,5$ & $1.863,7$ & $2.170,9$ & $1.802,2$ & $2.133,3$ & $2.803,5$ & 2.701, \\
\hline 2003 & $2.414,0$ & $2.249,3$ & $1.991,1$ & $1,547,9$ & $1.721,8$ & $1.654,8$ & $1.780,3$ & $1.592,5$ & $1.284,4$ & $1.227,8$ & $1.527,9$ & $1.678,7$ \\
\hline 2004 & $1.315,4$ & $1.058,7$ & $1.337,7$ & 997,1 & 882,6 & $1.347,1$ & $1.075,2$ & $1.098,7$ & $1.532,7$ & $1,695,6$ & $1.768,3$ & $1.796,6$ \\
\hline 2005 & $1.408,8$ & $1.433,8$ & $1,505,1$ & $1.718,5$ & $1,390,2$ & $1,371,9$ & $1,391,3$ & $1.274,3$ & $1.234,0$ & $1.105,7$ & $1.474,2$ & 1.859 \\
\hline
\end{tabular}

Fuente: Incopesca, 2011 
Revista de Política Económica y Desarrollo Sostenible EISSN: 2215-4167 • Vol. 2 (2) • Enero- Junio, 2017: 1-23

DOI: http://dx.doi.org/10.15359/peds.2-2.1

Moreno, Moya y Alfaro

URL: http://www.revistas.una.ac.cr/politicaeconomica

Anexo 2. Cantidad de turistas extranjeros en Costa Rica.

\begin{tabular}{rrr}
\hline Año & Total de Llegada de turistas & Visitantes de Soy y playa \\
\hline $\mathbf{1 9 9 0}$ & $435.037,00$ & $193.223,69$ \\
$\mathbf{1 9 9 1}$ & $504.649,00$ & $301.893,06$ \\
$\mathbf{1 9 9 2}$ & $610.093,00$ & $348.726,50$ \\
$\mathbf{1 9 9 3}$ & $684.005,00$ & $381.554,85$ \\
$\mathbf{1 9 9 4}$ & $761.448,00$ & $415.951,52$ \\
$\mathbf{1 9 9 5}$ & $784.616,00$ & $426.241,69$ \\
$\mathbf{1 9 9 6}$ & $781.127,00$ & $424.692,04$ \\
$\mathbf{1 9 9 7}$ & $811.490,00$ & $438.177,91$ \\
$\mathbf{1 9 9 8}$ & $942.778,00$ & $496.490,08$ \\
$\mathbf{1 9 9 9}$ & $1.031 .585,00$ & $535.934,12$ \\
$\mathbf{2 0 0 0}$ & $1.088 .075,00$ & $561.024,41$ \\
$\mathbf{2 0 0 1}$ & $1.131 .406,00$ & $580.270,07$ \\
$\mathbf{2 0 0 2}$ & $1.113 .359,00$ & $572.254,42$ \\
$\mathbf{2 0 0 3}$ & $1.237 .948,00$ & $627.591,20$ \\
$\mathbf{2 0 0 4}$ & $1.452 .926,00$ & $723.074,67$ \\
$\mathbf{2 0 0 5}$ & $1.679 .051,00$ & $823.509,13$ \\
\hline
\end{tabular}

Fuente: Elaboración propia con datos del ICT. 